\title{
Effect of Sodium Glucose Co-Transporter 2 Inhibitors on Liver Fat Mass and Body Composition in Patients with Nonalcoholic Fatty Liver Disease and Type 2 Diabetes Mellitus
}

\author{
Yoshitaka Arase ${ }^{1,2}$ (1) $\cdot$ Koichi Shiraishi $^{1,3} \cdot$ Kazuya Anzai $^{1,2} \cdot$ Hirohiko Sato $^{1,2} \cdot$ Erika Teramura $^{1,2} \cdot$ Kota Tsuruya $^{1}$. \\ Shunji Hirose ${ }^{1} \cdot$ Ryuzo Deguchi $^{1,2} \cdot$ Masao Toyoda $^{4} \cdot$ Tetsuya Mine $^{1} \cdot$ Tatehiro Kagawa $^{1}$
}

Published online: 16 April 2019

(c) The Author(s) 2019

\begin{abstract}
Background and Objective Sodium glucose co-transporter 2 inhibitors increase urinary glucose excretion and reduce visceral adiposity and body weight, but their efficacy on patients with nonalcoholic fatty liver disease has not been sufficiently investigated. The aim of this study was to assess the effect of sodium glucose co-transporter 2 inhibitors on liver fat mass and body composition in patients with nonalcoholic fatty liver disease and type 2 diabetes mellitus.

Methods We retrospectively analyzed 17 patients with nonalcoholic fatty liver disease and type 2 diabetes who received sodium glucose co-transporter 2 inhibitors between November 2016 and July 2017. Changes in liver fat, subcutaneous and visceral fat, body composition, and liver function-related parameters were assessed after 24 weeks of sodium glucose cotransporter 2 inhibitor treatment and compared to baseline values.

Results Ten patients received dapagliflozin at $5 \mathrm{mg} /$ day and seven patients received canagliflozin at $100 \mathrm{mg} /$ day for 24 weeks. All patients completed the study without any serious adverse effects and achieved body weight loss and improved glycated hemoglobin levels. Liver fat mass evaluated by proton magnetic resonance spectroscopy was significantly reduced (19.1\% vs. $9.2 \%, p<0.01$ ), and so were both subcutaneous and visceral fat mass. The body fat/body weight ratio decreased, whereas the skeletal muscle mass/body weight ratio increased. Liver function (aspartate aminotransferase, alanine aminotransferase, and $\gamma$-glutamyl transpeptidase) improved significantly.

Conclusions Sodium glucose co-transporter 2 inhibitor treatment not only improved glycemic control but also reduced liver fat mass in patients with nonalcoholic fatty liver disease and type 2 diabetes. Body weight loss was primarily attributable to a reduction in fat mass, especially visceral fat. Thus, sodium glucose co-transporter 2 inhibitors could potentially serve as a therapeutic agent for patients with nonalcoholic fatty liver disease and type 2 diabetes.
\end{abstract}

Yoshitaka Arase

arase@tokai-u.jp

1 Division of Gastroenterology and Hepatology, Department of Internal Medicine, Tokai University School of Medicine, 143 Shimokasuya, Isehara, Kanagawa 259-1193, Japan

2 Division of Gastroenterology and Hepatology, Tokai University Oiso Hospital, Nakagun, Kanagawa, Japan

3 Division of Gastroenterology and Hepatology, Tokai University Tokyo Hospital, Tokyo, Japan

4 Division of Nephrology, Endocrinology and Metabolism, Department of Internal Medicine, Tokai University School of Medicine, Isehara, Kanagawa, Japan

\section{Key Points}

Sodium glucose co-transporter 2 inhibitor treatment improved glycemic control and reduced liver fat mass in patients with nonalcoholic fatty liver disease and type 2 diabetes mellitus.

Body weight reduction was mostly due to a reduction in fat mass, especially visceral fat, rather than skeletal muscle mass.

Sodium glucose co-transporter 2 inhibitors may be highly useful therapeutic agents for patients with nonalcoholic fatty liver disease and type 2 diabetes. 


\section{Introduction}

Nonalcoholic fatty liver disease (NAFLD) is the most common chronic liver disease with an estimated prevalence rate of $25 \%$ worldwide [1]. Nonalcoholic fatty liver disease comprises a wide spectrum and ranges from nonalcoholic fatty liver, which rarely progresses to advanced liver diseases, to nonalcoholic steatohepatitis, which can lead to liver fibrosis and hepatocellular carcinoma [2, 3]. Previous studies identified a strong association between NAFLD and obesity, with a positive correlation between body mass index (BMI) and fatty liver [4]. Nonalcoholic fatty liver disease is considered a component of metabolic syndrome mediated by obesity-induced insulin resistance [5]. Furthermore, NAFLD is an independent risk factor for cardiovascular disease and chronic kidney disease [6-8] and is also significantly associated with the risk of incident type 2 diabetes mellitus (T2DM) [9, 10]. Many cross-sectional studies [11-13] demonstrated that T2DM is involved in the progression of liver fibrosis and hepatocellular carcinoma. Thus, proper management of T2DM is important to improve the prognosis of patients with NAFLD.

Sodium-glucose co-transporter 2 inhibitors (SGLT2i) suppress the reabsorption of glucose in the kidney and increase urinary glucose excretion, and thereby improve insulin resistance in patients with T2DM by decreasing blood glucose and body weight (BW) [14]. Furthermore, SGLT2i have been shown to reduce cardiovascular events and exert renal protective effects $[15,16]$. Recently, the benefits of SGLT2i treatment on liver function were reported in patients with NAFLD and T2DM [17-21], and the beneficial effects of SGLT2i on fatty liver in patients with T2DM were reviewed [22]. However, few studies have focused on changes in liver fat accumulation after SGLT2i treatment. In this study, we assessed the effect of SGLT2i treatment on liver fat accumulation in patients with NAFLD and T2DM by magnetic resonance imaging (MRI), and analyzed body composition and various parameters related to liver function.

\section{Methods}

\subsection{Study Design and Patient Population}

We retrospectively analyzed consecutive patients with NAFLD and T2DM who received SGLT2i monotherapy for more than 24 weeks at the Department of Gastroenterology and Hepatology, Tokai University Oiso Hospital (Kanagawa, Japan) between November 2016 and July 2017. These patients had not received anti-diabetic medication and did not respond to nutrition or exercise intervention for at least 24 weeks.

Nonalcoholic fatty liver disease was diagnosed based on imaging techniques [abdominal ultrasonography and/ or computed tomography (CT)] or liver biopsy (steatosis in 5\% or more of hepatocytes). The T2DM diagnosis was made according to the Report of the Expert Committee on the Diagnosis and Classification of Diabetes Mellitus [23]. Exclusion criteria were the presence of alcoholic liver diseases (alcohol consumption $>30 \mathrm{~g} /$ day for men and $20 \mathrm{~g} /$ day for women) [24], chronic viral hepatitis, autoimmune hepatitis, drug-induced liver injury, biliary tract disease, decompensated liver cirrhosis, hepatocellular carcinoma, history of diabetic ketoacidosis, active ongoing urinary tract infections, addition of other anti-diabetic drugs during the study period, and presence of severe comorbidities such as heart, respiratory, and/or renal dysfunction (estimated glomerular filtration rate $<45 \mathrm{~mL} / \mathrm{min} / 1.73 \mathrm{~m}^{2}$ ).

After SGLT2i treatment was started, patients were required to visit the outpatient clinic every 1 or 2 months. Patients received diet and exercise therapy instructions from a dietician every 2 months during SGLT2i treatment. These nutrition and exercise interventions were the same as those given prior to treatment. Adverse events were recorded on each visit and the severity assessed according to the Common Terminology Criteria for Adverse Events Version 4.0.

\subsection{Laboratory and Clinical Parameters}

We collected baseline information such as age, sex, BW, BMI (calculated as weight in $\mathrm{kg} / \mathrm{height}$ in $\mathrm{m}^{2}$ ), concomitant metabolic disease, and laboratory parameters. Diagnosis of metabolic diseases was based on defined characteristics and the patient's medication regimen. Hypertension was diagnosed in patients with systolic blood pressure $\geq 130 \mathrm{mmHg}$ and/or diastolic blood pressure $\geq 85 \mathrm{mmHg}$, or in those taking antihypertensive medication. Patients with serum triglyceride (TG) levels $\geq 150 \mathrm{mg} / \mathrm{dL}$ and/or high-density lipoprotein cholesterol (HDL-C) levels $<40 \mathrm{mg} / \mathrm{dL}$, or those taking lipid-lowering medication were diagnosed with dyslipidemia. Hyperuricemia was present in patients with serum uric acid levels $>7.0 \mathrm{mg} / \mathrm{dL}$, or in those taking uric acidlowering medication.

Blood samples were obtained in the outpatient clinic in the morning following a 12-h overnight fast. Laboratory parameters included blood cell counts and levels of serum aspartate aminotransferase (AST), alanine aminotransferase (ALT), $\gamma$-glutamyl transpeptidase, creatinine, blood urea nitrogen, uric acid, TG, total cholesterol, low-density lipoprotein cholesterol (LDL-C), HDL-C, glycated hemoglobin $\left(\mathrm{HbA}_{1 \mathrm{c}}\right)$, fasting plasma glucose, fasting insulin, and ferritin. $\mathrm{HbA}_{1 \mathrm{c}}$ was expressed as National Glycohemoglobin Standardization Program units (\%). Insulin resistance was 
evaluated by homeostasis model assessment-insulin resistance (HOMA-IR) using the following formula: HOMA$\mathrm{IR}=$ fasting insulin $(\mathrm{IU} / \mathrm{mL}) \times$ plasma glucose $(\mathrm{mg} / \mathrm{dL}) / 405$ [25]. Two non-invasive hepatic fibrosis scores were calculated according to the following formula: AST to platelet ratio index $($ APRI $)=[$ AST $(I U / L) / U L N(I U / L)] \times 100 /$ platelet count $\left(10^{9} / \mathrm{L}\right)$, where ULN is the upper limit of normal [26], and Fibrosis 4 (FIB-4) index $=[$ age $($ years $) \times$ AST $(\mathrm{IU} / \mathrm{L})] /\left[\right.$ platelet count $\left.\left(10^{9} / \mathrm{L}\right)\right] \times \sqrt{ }$ ALT $\left.(\mathrm{IU} / \mathrm{L})\right][27]$.

\subsection{Quantification of Liver Fat Mass}

Liver fat was determined by proton magnetic resonance spectroscopy $\left({ }^{1} \mathrm{H}-\mathrm{MRS}\right)$ [28-32]. Magnetic resonance imaging was performed using a $1.5 \mathrm{~T}$ unit (Signa HDxt Version 16, GE Healthcare, Milwaukee, WI, USA) with an eightchannel body array coil. For spectroscopy analysis, we used SAGE Version 7 (GE Healthcare). A $30 \times 30 \times 30 \mathrm{~mm}^{3}$ voxel was positioned in the liver right lobes with careful avoidance of ducts and vasculature. The power spectrum was calculated from the obtained magnetic resonance signal, and curve fitting was performed by the Lorenz curve. The area under the curve in the frequency range of water and fat was determined, and the fat/water ratio in the liver was calculated. The scanning parameters were as follows: pulse sequence: Probe-P, repetition time: $1149.0 \mathrm{~ms}$, echo time: $27.0 \mathrm{~ms}$, number of excitations: 2.00, acquisition matrix: $1 \times 1$, and scan time: $23 \mathrm{~s}$ with breath hold. Magnetic resonance imaging was conducted with the patient lying in the supine position. We started magnetic resonance spectroscopy imaging as a routine hepatic MRI sequence in August 2015, and performed this examination every 6 months to quantitatively evaluate liver fat in NAFLD patients.

\subsection{Evaluation of Body Composition}

Body composition was evaluated using InBody 770 (InBody Japan, Tokyo, Japan), a body composition analyzer equipped with simultaneous multi-frequency impedance measurement technology. In addition, the visceral fat area (VFA) and subcutaneous fat area (SFA) were analyzed by CT scans (Aquilion 128; Siemens Healthineers, Erlangen, Germany) using Fat Scan software (N2 System Corporation, Hyogo, Japan). A single cross-sectional scan at the level of the umbilicus (the fourth and fifth lumbar disc position) was selected for quantification $[33,34]$.

\subsection{Statistical Analysis}

The values obtained after the 24-week treatment were compared with baseline values using the Wilcoxon signed-rank test. We also evaluated the correlations between changes in ${ }^{1} \mathrm{H}$-MRS fat/water ratio and other variables using the
Table 1 Clinical characteristics of the patients in this study

\begin{tabular}{lc}
\hline Characteristics & Values \\
\hline Age (years) & $60(50-83)$ \\
Sex, female & $10(58.8)$ \\
Body weight $(\mathrm{kg})$ & $72.9(48.8-101.2)$ \\
BMI $\left(\mathrm{kg} / \mathrm{m}^{2}\right)$ & $28.2(25.1-36.8)$ \\
Hypertension & $7(41.2)$ \\
Dyslipidemia & $12(70.6)$ \\
Hyperuricemia & $9(52.9)$ \\
SGLT2i & \\
Dapagliflozin & $10(58.9)$ \\
Canagliflozin & $7(41.1)$ \\
\hline
\end{tabular}

Values are presented as median (range) or number (\%)

$B M I$ body mass index, $S G L T 2 i$ sodium glucose co-transporter 2 inhibitor

Spearman coefficient. All analyses were performed using SPSS Version 24 (SPSS Japan, Tokyo, Japan), and $p$ values $<0.05$ were considered statistically significant.

\subsection{Ethical Approval}

This study was approved by the Institutional Review Board for Clinical Research at Tokai University (No. 17R-376). All procedures performed in studies involving human participants were in accordance with the ethical standards of the institutional research committee and with the 1964 Helsinki Declaration and its later amendments or comparable ethical standards.

\section{Results}

\subsection{Patients' Characteristics}

Seventeen patients were included in this study, and their clinical characteristics are presented in Table 1. The median patient age was 60 years (range $50-83$ years), and ten patients $(58.8 \%)$ were female. All patients had a BMI of $25 \mathrm{~kg} / \mathrm{m}^{2}$ or more. This cohort included seven patients with hypertension (41.2\%), 12 with dyslipidemia (70.6\%), and nine with hyperuricemia (52.9\%). Ten patients received dapagliflozin at a dose of $5 \mathrm{mg} /$ day and seven patients received canagliflozin at a dose of $100 \mathrm{mg} /$ day for 24 weeks.

\subsection{Effect of Sodium-Glucose Co-Transporter 2 Inhibitors on Liver Fat Mass and Body Composition}

Proton magnetic resonance spectroscopy revealed a significant reduction in the liver fat/water ratio from $19.1 \%$ 


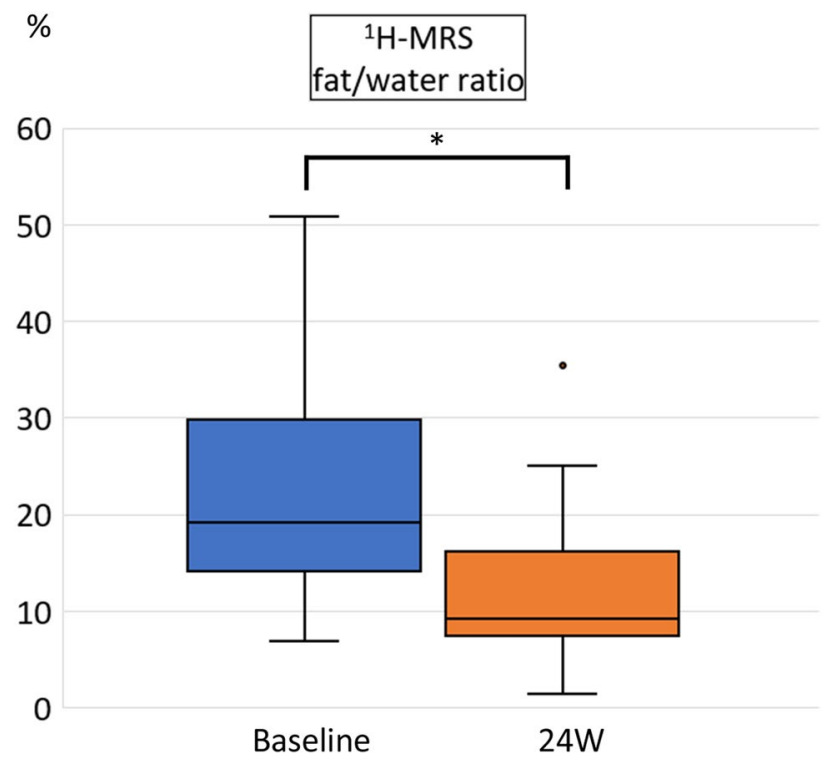

Fig. 1 Hepatic fat/water ratio measured by proton magnetic resonance spectroscopy $\left({ }^{1} \mathrm{H}-\mathrm{MRS}\right)$ before and after 24 weeks (W) of sodium glucose co-transporter 2 inhibitor treatment. Data are shown as boxand-whisker plots and expressed as a median with the interquartile range and maximum and minimum values. The dot represents an outlier defined as the value exceeding the 75th percentile plus 1.5 times the interquartile range. ${ }^{*} p<0.01$ (Wilcoxon signed-rank test)

(median, range 6.9-50.7\%) at baseline to $9.2 \%$ (range $1.3-5.3 \%)$ in week $24(p<0.001$; Fig. 1). Sodium-glucose co-transporter 2 inhibitor treatment significantly

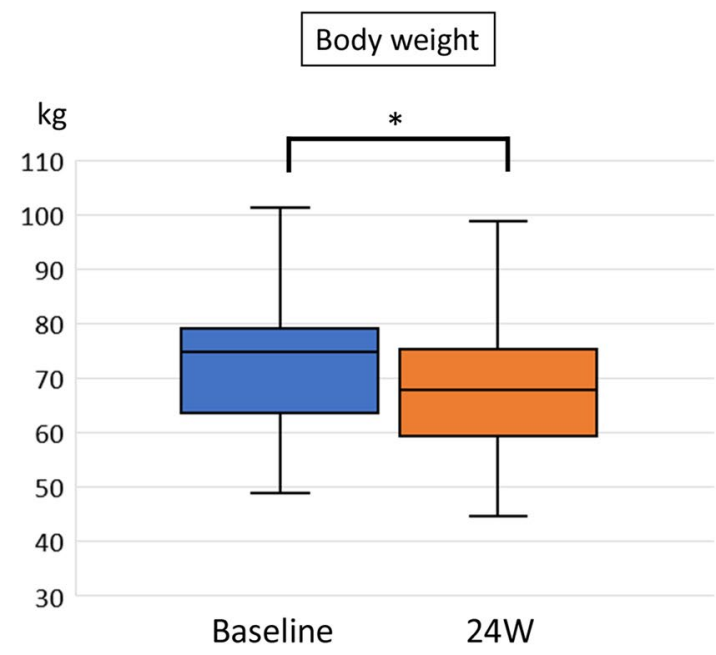

Fig. 2 Body weight and body mass index (BMI) before and after 24 weeks $(\mathrm{W})$ of sodium glucose co-transporter 2 inhibitor treatment. Data are presented as box-and-whisker plots and expressed as a median with the interquartile range and maximum and minimum reduced both BW [72.9 kg (median, range 48.8-101.2 kg) vs. $67.7 \mathrm{~kg}$ (median, range $44.7-98.7 \mathrm{~kg}$ ), $p<0.001]$ and BMI $\left[28.2 \mathrm{~kg} / \mathrm{m}^{2}\right.$ (median, range $25.1-36.8$ ) vs. $27.2 \mathrm{~kg} /$ $\mathrm{m}^{2}$ (median, range 21.7-35.0), $p<0.001$ ] (Fig. 2). Notably, every patient lost weight throughout the course of the study.

We next analyzed the effect of SGLT2i on body composition (Fig. 3). The skeletal muscle mass/BW ratio significantly increased from $34.8 \%$ (median, range $25.0-45.5 \%$ ) to $36.5 \%$ (median, range $28.9-47.2 \%, p=0.001$ ), whereas the body fat/BW ratio significantly decreased from $35.4 \%$ (median, range 20.1-52.6) to $34.1 \%$ (median, range $19.6-50.8, p=0.002)$. Significant increases in the total body water/weight ratio $(47.4-49.3 \% ; p=0.002)$ and the skeletal muscle mass/body fat mass ratio (SF ratio) $(0.98-1.10 ; p=0.001)$ were also observed. There were no marked changes in protein and soft lean mass during the treatment period (data not shown).

We evaluated changes in VFA and SFA by abdominal CT scans (Fig. 4). Both fat areas were significantly reduced, with VFA declining from $139 \mathrm{~cm}^{2}$ (median, range $89-314 \mathrm{~cm}^{2}$ ) to $121 \mathrm{~cm}^{2}$ (median, range $78-320 \mathrm{~cm}^{2}$, $p=0.001$ ) and SFA from $222 \mathrm{~cm}^{2}$ (median, range $112-449 \mathrm{~cm}^{2}$ ) to $214 \mathrm{~cm}^{2}$ (median, range $101-332 \mathrm{~cm}^{2}$, $p=0.002)$. Visceral fat area reduction was greater than that of SFA ( $14.7 \%$ vs. $7.4 \% ; p=0.005)$, and there was a significant correlation between changes in VFA and BW (correlation coefficient $=0.711 ; p=0.001$ ).

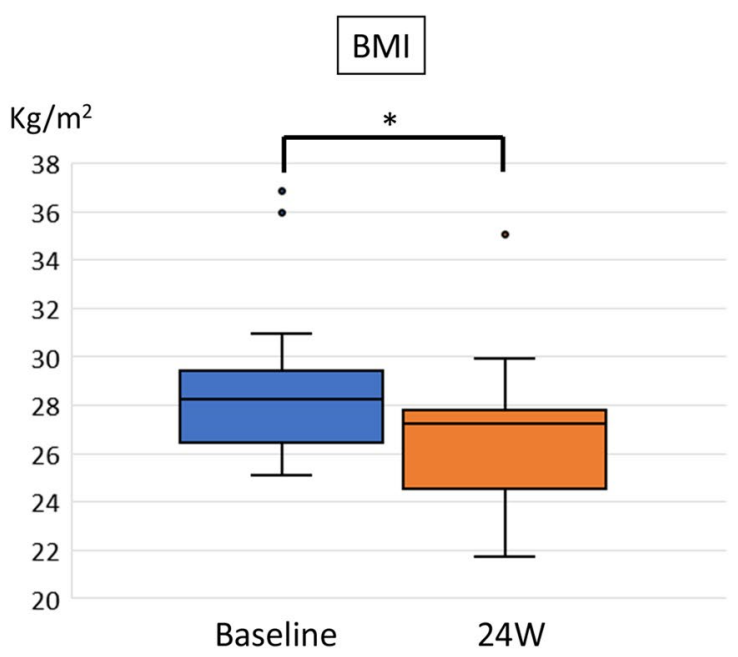

values. Dots represent outliers defined as values exceeding the 75 th percentile plus 1.5 times the interquartile range. ${ }^{*} p<0.01$ (Wilcoxon signed-rank test) 

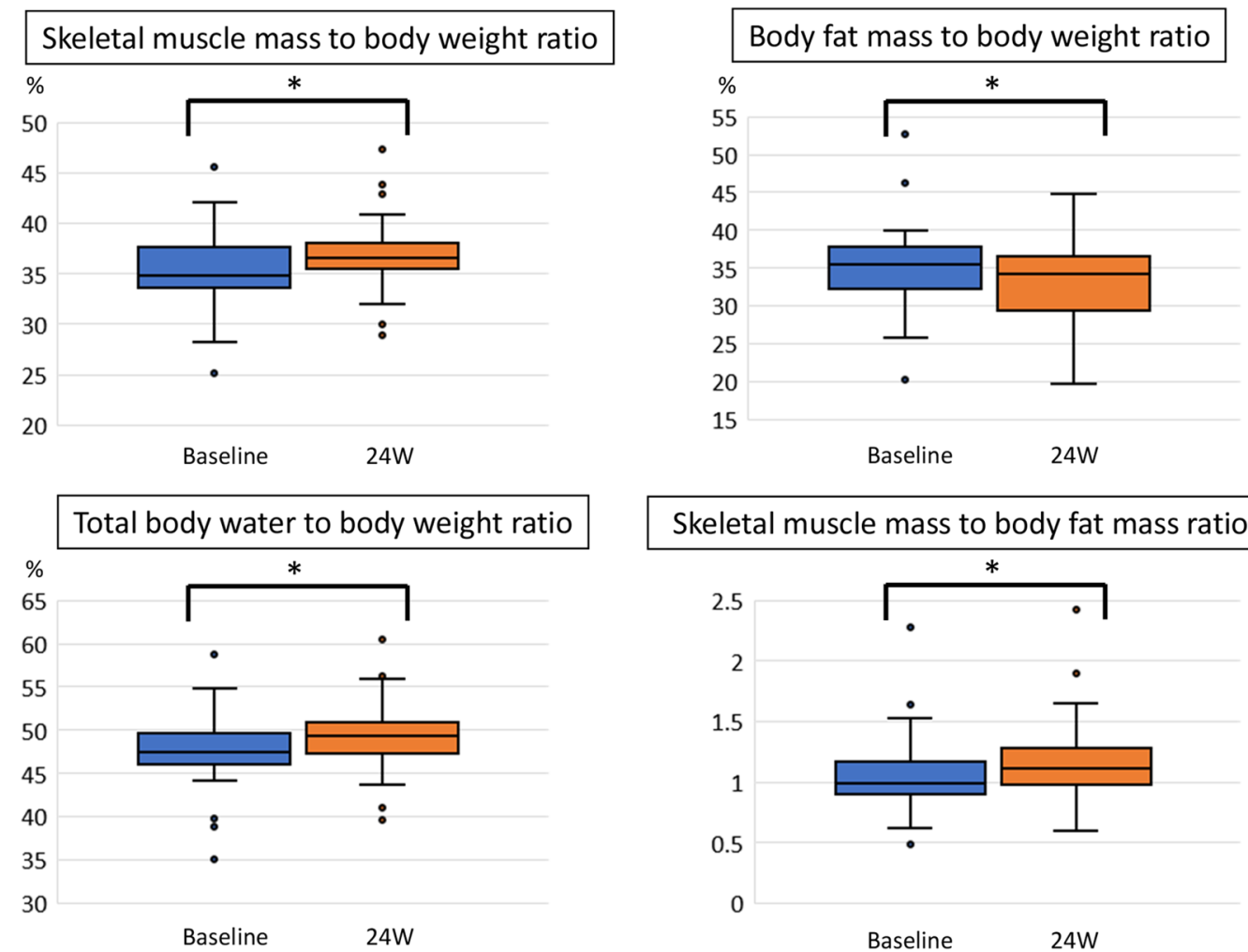

Skeletal muscle mass to body fat mass ratio (SF ratio)

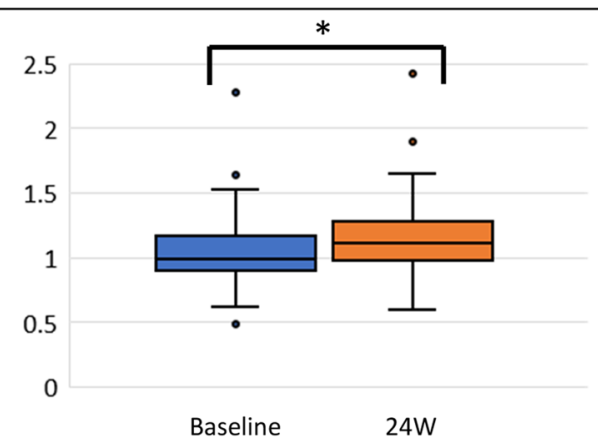

Fig. 3 Skeletal muscle mass to body weight ratio, body fat mass to body weight ratio, total body water to body weight ratio, and skeletal muscle mass to body fat mass ratio (SF ratio) before and after 24 weeks (W) of sodium glucose co-transporter 2 inhibitor treatment. Data are presented as box-and-whisker plots and expressed as

a median with the interquartile range and maximum and minimum values. Dots represent outliers defined as values exceeding the 75 th percentile plus 1.5 times the interquartile range. $* p<0.01$ (Wilcoxon signed-rank test)

\section{visceral fat area}

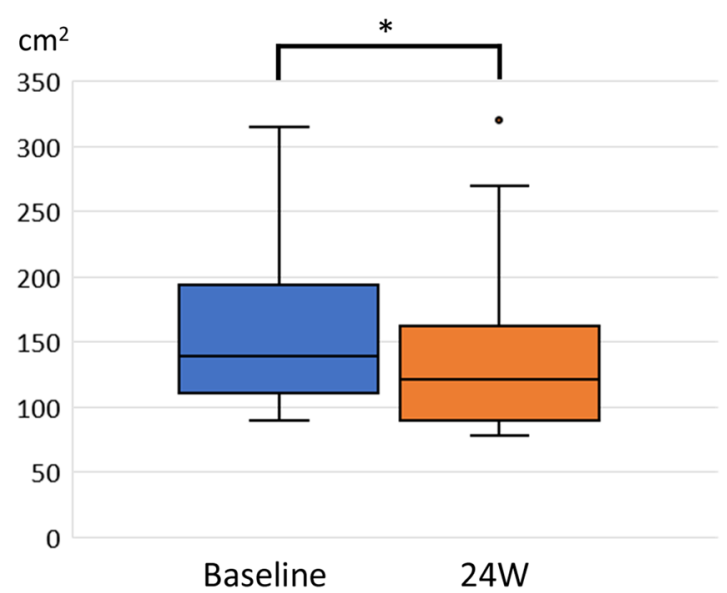

Fig. 4 Visceral and subcutaneous fat areas measured by computed tomography scans before and after 24 weeks (W) of sodium glucose co-transporter 2 inhibitors treatment. Data are presented as box-andwhisker plots and expressed as a median with the interquartile range

\section{subcutaneous fat area}

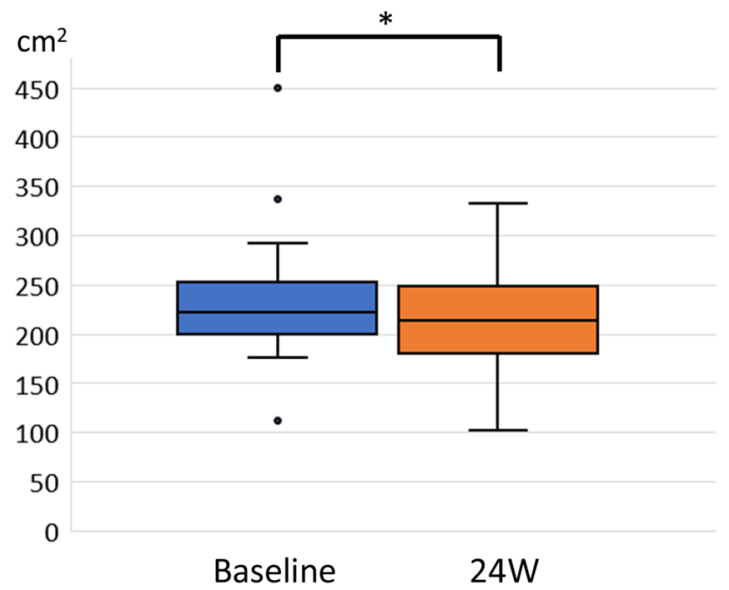

and maximum and minimum values. Dots represent outliers defined as values exceeding the 75 th percentile plus 1.5 times the interquartile range. ${ }^{*} p<0.01$ (Wilcoxon signed-rank test) 
Table 2 Changes in laboratory findings at baseline and after 24 weeks of SGLT2i treatment in patients with NAFLD and type 2 diabetes mellitus

\begin{tabular}{|c|c|c|c|}
\hline Parameter & Baseline & Week 24 & $p$ value \\
\hline $\mathrm{HbA}_{1 \mathrm{c}}(\%)$ & $6.5(6.3-7.7)$ & $5.9(5.4-6.8)$ & 0.003 \\
\hline FPG $(\mathrm{mg} / \mathrm{dL})$ & $132(127-185)$ & $112(89-144)$ & $<0.001$ \\
\hline $\begin{array}{l}\text { Fasting insulin }(\mu \mathrm{U} / \\
\mathrm{mL})\end{array}$ & $18.5(5-83.9)$ & $11.8(1.6-21.0)$ & $<0.001$ \\
\hline HOMA-IR & $6.3(2.0-27.3)$ & $3.2(0.4-6.2)$ & $<0.001$ \\
\hline $\begin{array}{l}\text { Platelet count }\left(\times 10^{4} /\right. \\
\mu \mathrm{L})\end{array}$ & $20.9(12.6-38.3)$ & $20.5(12.7-33.1)$ & 0.298 \\
\hline AST (IU/L) & $30(15-83)$ & $21(10$ & 0.007 \\
\hline ALT (IU/L) & $48(14-118)$ & $27(12-96)$ & 0.005 \\
\hline GGT (IU/L) & $42(22-124)$ & $28(12-87)$ & 0.001 \\
\hline Ferritin (ng/dL) & 130 & 292) & 0.002 \\
\hline FIB-4 index & $1.42(0.54-4.30)$ & $1.28(0.53-3.14)$ & 0.163 \\
\hline APRI & $0.43(0.24-2.05)$ & $0.35(0.14-1.44)$ & 0.031 \\
\hline Creatinine $(\mathrm{mg} / \mathrm{dL})$ & $0.76(0.55-1.17)$ & $0.82(0.60-1.11)$ & 0.023 \\
\hline BUN (mg/dL) & $13(9-20)$ & $15(8-21)$ & 0.082 \\
\hline $\begin{array}{l}\mathrm{eGFR}(\mathrm{mL} / \\
\left.\mathrm{min} / 1.73 \mathrm{~m}^{2}\right)\end{array}$ & $66(49-86)$ & $64(49-81)$ & 0.108 \\
\hline$(\mathrm{mg} / \mathrm{dL})$ & $5.7(2$ & $7-8.0)$ & 0.016 \\
\hline $\mathrm{TG}(\mathrm{mg} / \mathrm{dL})$ & $154(70-300)$ & $119(57-224)$ & 0.039 \\
\hline Total-C (mg/dL) & $190(147-234)$ & $185(145-228)$ & 0.733 \\
\hline LDL-C (mg/dL) & $122(85-149)$ & $112(86-151)$ & 0.776 \\
\hline HDL-C (mg/dL) & $47(34-85)$ & $49(42-80)$ & 0.14 \\
\hline TG/HDL-C ratio & $3.0(1.1-7.5)$ & $2.2(0.8-5.3)$ & 0.015 \\
\hline
\end{tabular}

Values are presented as median (range)

$A L T$ alanine aminotransferase, APRI AST to platelet ratio index, $A S T$ aspartate aminotransferase, $B U N$ blood urea nitrogen, $e G F R$ estimated glomerular filtration rate, FIB-4 Fibrosis-4, FPG fasting plasma glucose, $G G T \gamma$-glutamyl transferase, $H b A_{I c}$ glycated hemoglobin, $H D L-C$ high-density lipoprotein cholesterol, HOMA-IR homeostasis model assessment-insulin resistance, $L D L-C$ low-density lipoprotein cholesterol, $T G$ triglyceride, Total-C total cholesterol

\subsection{Effect of Sodium-Glucose Co-Transporter 2 Inhibitors on Clinical Parameters}

The 24-week SGLT2i treatment significantly improved glucose metabolism-related variables including $\mathrm{HbA}_{1 \mathrm{c}}$ (median: 6.5 vs. $5.9 \%, p=0.003$ ), fasting plasma glucose (median: 132 vs. $112 \mathrm{mg} / \mathrm{dL}, p<0.001$ ), fasting insulin (median: 18.5 vs. $11.8 \mu \mathrm{U} / \mathrm{mL}, p<0.001$ ), and HOMA-IR (median 6.3-3.2, $p<0.001$; Table 2). Serum AST (median: 30 vs. $21 \mathrm{IU} / \mathrm{L}$ ), ALT (median: 48 vs. 27 IU/L), and $\gamma$-glutamyl transpeptidase (median: 42 vs. 28 IU/L) levels also declined significantly. Analysis of liver fibrosis-related variables revealed that SGLT2i treatment significantly decreased the APRI (median 0.43 vs. $0.35, p=0.031$ ) but not the FIB-4 index. Serum creatinine slightly increased, but there were no significant changes in blood urea nitrogen and estimated glomerular filtration rate levels. Serum ferritin (median: 130-77 ng/
$\mathrm{dL}, p=0.002$ ), TG (median: $154-119 \mathrm{mg} / \mathrm{dL}, p=0.039$ ), uric acid (median: $5.7-4.9 \mathrm{mg} / \mathrm{dL}, p=0.016$ ), and the TG/ HDL-C ratio (median: $3.0-2.2, p=0.015$ ) were significantly improved by SGLT2i treatment. Reduction in ${ }^{1} \mathrm{H}-\mathrm{MRS}$ reflecting changes in liver fat correlated significantly with changes in BW (correlation coefficient $=0.505, p=0.039$ ), fasting insulin (correlation coefficient $=0.642, p=0.005$ ), HOMA-IR (correlation coefficient $=0.620, p=0.008$ ), ferritin (correlation coefficient $=0.520, p=0.033$ ), and body fat mass (correlation coefficient $=0.635, p=0.006$ ).

\subsection{Safety}

Mild adverse events observed during 24 weeks of SGLT2i treatment included itching of the genital area in two patients $(11.8 \%)$ and urinary tract infection in one patient (5.9\%). There were no serious adverse events.

\section{Discussion}

In this study, we demonstrated that SGLT2i treatment not only reduced BW but also improved liver fat accumulation in patients with NAFLD and T2DM. The observed weight loss was mostly due to a reduction in body fat mass rather than skeletal muscle mass.

Deposition of fat in the liver occurs as a consequence of increased free fatty acid influx from food or fat cells, increased de novo lipid synthesis in the liver, or decreased free fatty acid oxidation and TG trafficking [35]. In clinical practice, abdominal ultrasonography is generally used to diagnose fatty liver. Nevertheless, this imaging technique has low sensitivity and low reproducibility in the quantification of liver fat [36], and the diagnosis may be influenced by the skill level of the operator. Although liver biopsy was regarded as the gold standard for the diagnosis and histological assessment of NAFLD, it has several limitations such as sampling error, invasiveness, and inter-observer differences [37]. The liver-to-spleen ratio may be obtained by a CT scan to quantify liver fat accumulation [38], but its disadvantages include radiation exposure and inadequate sensitivity to detect minor steatosis and slight changes in fat content [39]. An ideal device to monitor liver fat content should be non-invasive, safe, sensitive, accurate, and repeatable, which renders MRI the method of choice [40]. Proton magnetic resonance spectroscopy can accurately determine the fat/water ratio in hepatic tissue by measuring the chemical shift of protons in water and lipids [31, 41]. In fact, results obtained by ${ }^{1} \mathrm{H}-\mathrm{MRS}$ were proven to be highly correlated with actual hepatic steatosis $[29,30]$. The present study demonstrated significant a reduction in liver fat mass evaluated by ${ }^{1} \mathrm{H}$-MRS after 24 weeks of SGLT2i treatment in patients with NAFLD and T2DM. These results 
are in agreement with two previous studies using MRI. The E-LIFT trial [42] conducted in India showed a significant reduction in liver fat mass evaluated by MRI-proton density fat fraction after 20 weeks of empagliflozin treatment, and the LEAD trial [43] conducted in Japan demonstrated a significant reduction in liver fat mass evaluated by MRI hepatic fat fraction after 24 weeks of luseogliflozin treatment. In our study, liver fat mass reduction was significantly correlated with BW reduction, which is consistent with the results of the LEAD trial. Changes in serum ferritin, fasting insulin, HOMA-IR, and body fat mass were also significantly correlated with liver fat mass reduction.

In contrast to SGLT2i, sitagliptin, an oral antidiabetic drug that inhibits dipeptidyl peptidase IV, did not improve liver fat mass evaluated by MRI-proton density fat fraction after 24 weeks of treatment [44, 45]. A recent meta-analysis showed that treatment with another antidiabetic drug, metformin (a biguanide agent), did also not achieve a reduction in liver fat mass [46]. Pioglitazone reduced liver fat mass [47] but was associated with a risk of BW gain. However, glucagon-like peptide-1 receptor agonists have been reported to reducing liver fat content and BW [48-50], similar to SGLT2i.

In this study, the ratio between skeletal muscle mass and BW significantly increased, whereas the body fat mass to BW ratio significantly decreased with 24 weeks of SGLT2i treatment. Decreasing body fat mass without a loss of skeletal muscle mass is an important aspect of NAFLD treatment, which is why the relationship between sarcopenia (characterized by a decrease in skeletal muscle mass and muscle strength [51]) and NAFLD has gained much attention. Approximately $12 \%$ of patients with NAFLD are diagnosed with sarcopenia [52]. In a Korean study, even $22.7 \%$ of patients with NAFLD were identified with sarcopenia and it was an independent risk factor for nonalcoholic steatohepatitis and significant fibrosis [53]. Mizuno et al. [54] reported that the reduction in serum ALT levels within 12 months was significantly greater in patients with an increased SF ratio compared with those with a decreased SF ratio. The precise mechanisms are unknown, but changes in adipokines, myokines, and hepatokines may be involved. In our study, SGLT2i administration increased both skeletal muscle percentage and the SF ratio, suggesting that SGLT2i does not induce sarcopenia.

Baseline median VFA was $139 \mathrm{~cm}^{2}$ in this study, exceeding the cut-off value of $100 \mathrm{~cm}^{2}$ that indicates visceral obesity based on the proposed definition of the Japan Society for the Study of Obesity. It decreased by $12.8 \%$ after 24 weeks of SGLT2i treatment. Only a few reports have demonstrated a decrease in visceral fat caused by SGLT2i [55-57]. Recently, results of SGLT2 inhibitors on body composition and metabolic parameters in a Japanese population have been reported (LIGHT Study); luseogliflozin 52-week treatment resulted in body fat reduction, and minimal muscle and bone mineral content reductions in moderately obese Japanese patients with T2DM [57]. The changes in body content by SGLT2i were similar to our study, and it was also consistent that the changes of VFA and BW were significantly correlated. The excess accumulation of visceral fat is linked to the onset of arteriosclerotic diseases such as T2DM, dyslipidemia, and hypertension [58-60]. Decreasing visceral fat mass improves insulin resistance, hypertension, and dyslipidemia, and hence reduces the risk of cardiovascular diseases [61, 62].

In this study, serum TG levels, uric acid levels, and the TG/HDL-C ratio decreased significantly. The decrease in TG appears to be a result of improved insulin resistance because of diminished VFA [63]. Maruyama et al. [64] reported a correlation between the TG/HDL-C ratio and small dense LDL-C levels, which are associated with the occurrence of cardiovascular events [65]. The decrease in the TG/HDL-C ratio observed in our study may reflect lower small dense LDL-C levels, which would be in agreement with a previous study using dapagliflozin [66]. Increased serum uric acid levels are a known risk factor for cardiovascular events [67]. Sodium glucose co-transporter 2 inhibitor treatment decreased uric acid levels by mechanisms yet unknown. Sodium glucose co-transporter 2 inhibitors may promote uric acid excretion into the urine via glucose transporter 9 activation by inhibiting SGLT2-mediated glucose reabsorption [68, 69]. Decreased serum TG levels, uric acid levels, and the TG/HDL-C ratio resulting from SGLT2i treatment may lead to the prevention of future cardiovascular events.

There are currently six types of SGLT2i-based drugs on the market in Japan. Although some of these drugs (e.g., canagliflozin and sotagliflozin) exert mild or moderate inhibitory effects on intestinal and renal SGLT1 as well [70], there are no documented differences in the clinical effect of the available SGLT2i-based drugs [71]. We did not observe any obvious differences between the two drugs we used in our study (dapagliflozin and canagliflozin) with regard to the improvement of liver fat mass, BW, and various metabolic pathways.

A recent study showed that a BW loss of at least 7\% was necessary to improve the NAFLD Activity Score, a histological scoring system composed of hepatic steatosis, lobular inflammation, and ballooning degeneration [47, 72]. In our study, SGLT2i treatment for 24 weeks resulted in a $9.4 \%$ BW reduction and hence might improve the NAS; however, we did not perform any liver biopsies.

In patients with NAFLD, liver fibrosis was identified as the most important factor contributing to liver-related death [73]. Furthermore, T2DM is a significant risk factor for advanced liver fibrosis [74]. It is therefore important to analyze whether SGLT2i treatment can improve liver fibrosis. In this study, the APRI but not the FIB-4 index improved 
significantly. Although reports have indicated that SGLT2i treatment significantly reduces the FIB-4 index after a follow up of 24 weeks [75], 26 weeks [76], and 48 weeks [77], other reports found no significant reduction in the FIB-4 index $[43,78]$, showing that there is no consensus. No studies have reported significant reductions in APRI with SGLT2i treatment. Because we had not performed liver biopsies after the 24-week SGLT2i treatment, we could not accurately assess whether there were improvements in histologic fibrosis. Akuta et al. [79] reported that 24 weeks of treatment with canagliflozin in patients with NAFLD and T2DM achieved histological improvement; scores of the fibrosis stage decreased by $33 \%$ compared with the pretreatment. However, long-term observations are necessary to determine whether SGLT2i improves liver fibrosis in patients with NAFLD and T2DM.

Some patients experienced itching of the genital area $(11.8 \%)$ and urinary tract infection (5.9\%) during SGLT2i treatment. These known adverse effects were mild and did not interrupt treatment. Sodium glucose co-transporter 2 inhibitors therefore appeared sufficiently safe.

Our study has some limitations. We included only a small number of patients and lacked a placebo group, which may limit the strength of our conclusions. Furthermore, this was a retrospective study and we cannot completely exclude the possibility of selection bias. In addition, the treatment period was comparatively short. Nevertheless, our study suggests potential benefits and safety of SGLT2i treatment in patients with NAFLD and T2DM. Further large-scale and long-term prospective studies are warranted to confirm our results.

\section{Conclusion}

In this study, we showed that SGLT2i treatment not only improved glycemic control but also reduced liver fat mass in patients with NAFLD and T2DM. Importantly, BW reduction was mostly due to a reduction in fat mass, especially visceral fat, rather than skeletal muscle mass. Thus, SGLT2i are highly useful therapeutic agents for patients with NAFLD and T2DM.

Acknowledgements We thank Yuhei Otsuka at the Department of Radiology, Tokai University Oiso Hospital, Kanagawa, Japan for technical support with ${ }^{1} \mathrm{H}-\mathrm{MRS}$. We also thank Editage (http://www.edita ge.jp) for English language editing.

Author Contributions YA, KS, KA, HS, ET, and RD planned this study's content, collected patient's data, and wrote the manuscript. KT, SH, MT, TM, and TK provided technical or material support. YA drafted the manuscript. All authors analyzed the data, reviewed the manuscript, and approved the final version for publication.

\section{Compliance with Ethical Standards}

Funding No sources of funding were received for this article.

Conflict of interest Yoshitaka Arase, Koichi Shiraishi, Kazuya Anzai, Hirohiko Sato, Erika Teramura, Kota Tsuruya, Shunji Hirose, Ryuzo Deguchi, Masao Toyoda, Tetsuya Mine, and Tatehiro Kagawa have no conflicts of interest that are directly relevant to the content of this article.

Ethics approval All procedures performed in studies involving human participants were in accordance with the ethical standards of the institutional research committee and with the 1964 Helsinki Declaration and its later amendments or comparable ethical standards. This study was approved by the Institutional Review Board for Clinical Research at Tokai University (No. 17R-376).

Informed Consent As this was a retrospective study, formal consent was not required.

Data availability All data generated or analyzed during this study are included in this published article.

Open Access This article is distributed under the terms of the Creative Commons Attribution-NonCommercial 4.0 International License (http://creativecommons.org/licenses/by-nc/4.0/), which permits any noncommercial use, distribution, and reproduction in any medium, provided you give appropriate credit to the original author(s) and the source, provide a link to the Creative Commons license, and indicate if changes were made.

\section{References}

1. Younossi ZM, Koenig AB, Abdelatif D, Fazel Y, Henry L, Wymer M. Global epidemiology of nonalcoholic fatty liver disease: metaanalytic assessment of prevalence, incidence, and outcomes. Hepatology. 2016;64:73-84.

2. Chalasani N, Younossi Z, Lavine JE, Diehl AM, Brunt EM, Cusi $\mathrm{K}$, et al. The diagnosis and management of non-alcoholic fatty liver disease: practice guideline by the American Association for the Study of Liver Diseases, American College of Gastroenterology, and the American Gastroenterological Association. Hepatology. 2012;55:2005-23.

3. Goldberg D, Ditah IC, Saeian K, Lalehzari M, Aronsohn A, Gorospe EC, et al. Changes in the prevalence of hepatitis $\mathrm{C}$ virus infection, nonalcoholic steatohepatitis, and alcoholic liver disease among patients with cirrhosis or liver failure on the waitlist for liver transplantation. Gastroenterology. 2017;152:1090-9.

4. Eguchi Y, Hyogo H, Ono M, Mizuta T, Ono N, Fujimoto K, et al. Prevalence and associated metabolic factors of nonalcoholic fatty liver disease in the general population from 2009 to 2010 in Japan: a multicenter large retrospective study. J Gastroenterol. 2012;47(5):586-95.

5. Marchesini G, Brizi M, Morselli-Labate AM, Bianchi G, Bugianesi E, McCullough AJ, et al. Association of nonalcoholic fatty liver disease with insulin resistance. Am J Med. 1999;107:450-5.

6. Targher G, Byrne CD, Lonardo A, Zoppini G, Barbui C. Nonalcoholic fatty liver disease and risk of incident cardiovascular disease: a meta-analysis. J Hepatol. 2016;65:589-600.

7. Arase Y, Suzuki F, Kobayashi M, Suzuki Y, Kawamura Y, Matsumoto $\mathrm{N}$, et al. The development of chronic kidney disease in 
Japanese patients with nonalcoholic fatty liver disease. Intern Med. 2011;50:1081-7.

8. Mantovani A, Zaza G, Byrne CD, Lonardo A, Zoppini G, Bonora E, et al. Nonalcoholic fatty liver disease increases risk of incident chronic kidney disease: a systematic review and meta-analysis. Metabolism. 2018;79:64-76.

9. Vernon G, Baranova A, Younossi ZM. Systematic review: the epidemiology and natural history of non-alcoholic fatty liver disease and non-alcoholic steatohepatitis in adults. Aliment Pharmacol Ther. 2011;34:274-85.

10. Mantovani A, Byrne CD, Bonora E, Targher G. Nonalcoholic fatty liver disease and risk of incident type 2 diabetes: a meta-analysis. Diabetes Care. 2018;41:372-82.

11. Angulo P, Keach JC, Batts KP, Lindor KD. Independent predictors of liver fibrosis in patients with nonalcoholic steatohepatitis. Hepatology. 1999;30(6):1356-62.

12. Hossain N, Afendy A, Stepanova M, Nader F, Srishord M, Rafiq N, et al. Independent predictors of fibrosis in patients with nonalcoholic fatty liver disease. Clin Gastroenterol Hepatol. 2009;7(11):1224-9.

13. Yasui K, Hashimoto E, Komorizono Y, Koike K, Arii S, Imai Y, Japan NASH Study Group, Ministry of Health, Labour, and Welfare of Japan, et al. Characteristics of patients with nonalcoholic steatohepatitis who develop hepatocellular carcinoma. Clin Gastroenterol Hepatol. 2011;9(5):428-33.

14. Yokono M, Takasu T, Hayashizaki Y, Mitsuoka K, Kihara R, Muramatsu Y, et al. SGLT2 selective inhibitor ipragliflozin reduces body fat mass by increasing fatty acid oxidation in highfat diet-induced obese rats. Eur J Pharmacol. 2014;727:66-74.

15. Zinman B, Wanner C, Lachin JM, Fitchett D, Bluhmki E, Hantel S, EMPA-REG OUTCOME investigators, et al. Empagliflozin, cardiovascular outcomes, and mortality in type 2 diabetes. N Engl J Med. 2015;373:2117-28.

16. Neal B, Perkovic V, Mahaffey KW, de Zeeuw D, Fulcher G, Erondu N, CANVAS Program Collaborative Group, et al. Canagliflozin and cardiovascular and renal events in type 2 diabetes. N Engl J Med. 2017;377:644-57.

17. Leiter LA, Forst T, Polidori D, Balis DA, Xie J, Sha S. Effect of canagliflozin on liver function tests in patients with type 2 diabetes. Diabetes Metab. 2016;42:25-32.

18. Seko Y, Sumida Y, Tanaka S, Mori K, Taketani H, Ishiba H, et al. Effect of sodium glucose cotransporter 2 inhibitor on liver function tests in Japanese patients with non-alcoholic fatty liver disease and type 2 diabetes mellitus. Hepatol Res. 2017;47:1072-8.

19. Seko Y, Sumida $Y$, Sasaki K, Itoh Y, Iijima H, Hashimoto T, et al. Effects of canagliflozin, an SGLT2 inhibitor, on hepatic function in Japanese patients with type 2 diabetes mellitus: pooled and subgroup analyses of clinical trials. J Gastroenterol. 2018;53:140-51.

20. Tobita H, Sato S, Miyake T, Ishihara S, Kinoshita Y. Effects of dapagliflozin on body composition and liver tests in patients with nonalcoholic steatohepatitis associated with type 2 diabetes mellitus: a prospective, open-label, uncontrolled study. Curr Ther Res Clin Exp. 2017;87:13-9.

21. Akuta N, Watanabe C, Kawamura Y, Arase Y, Saitoh S, Fujiyama $\mathrm{S}$, et al. Effects of a sodium-glucose cotransporter 2 inhibitor in nonalcoholic fatty liver disease complicated by diabetes mellitus: preliminary prospective study based on serial liver biopsies. Hepatol Commun. 2017;1:46-52.

22. Scheen AJ. Beneficial effects of SGLT2 inhibitors on fatty liver in type 2 diabetes: a common comorbidity associated with severe complications. Diabetes Metab. 2019. https://doi.org/10.1016/j. diabet.2019.01.008.

23. Committee of the Japan Diabetes Society on the Diagnostic Criteria of Diabetes Mellitus, Seino Y, Nanjo K, Tajima N, Kadowaki T, Kashiwagi A, et al. Report of the committee on the classification and diagnostic criteria of diabetes mellitus. J Diabetes Investig. 2010;1:212-28.

24. Bellentani S, Saccoccio G, Costa G, Tiribelli C, Manenti F, Sodde M. Drinking habits as cofactors of risk for alcohol induced liver damage. The Dionysos Study Group. Gut. 1997;41:845-50.

25. Matthews DR, Hosker JP, Rudenski AS, Naylor BA, Treacher DF, Turner RC. Homeostasis model assessment: insulin resistance and beta-cell function from fasting plasma glucose and insulin concentrations in man. Diabetologia. 1985;28:412-9.

26. Wai CT, Greenson JK, Fontana RJ, Kalbfleisch JD, Marrero JA, Conjeevaram HS, et al. A simple noninvasive index can predict both significant fibrosis and cirrhosis in patients with chronic hepatitis C. Hepatology. 2003;38:518-26.

27. Shah AG, Lydecker A, Murray K, Tetri BN, Contos MJ, Sanyal AJ, Nash Clinical Research Network. Comparison of noninvasive markers of fibrosis in patients with nonalcoholic fatty liver disease. Clin Gastroenterol Hepatol. 2009;7:1104-12.

28. Cowin GJ, Jonsson JR, Bauer JD, Ash S, Ali A, Osland EJ, et al. Magnetic resonance imaging and spectroscopy for monitoring liver steatotis. J Magn Reson Imaging. 2008;28:937-45.

29. Keese D, Korkusuz H, Huebner F, Namgaladze D, Raschidi B, Vogl TJ. In vivo and ex vivo measurements: noninvasive assessment of alcoholic fatty liver using $1 \mathrm{H}-\mathrm{MR}$ spectroscopy. Diagn Interv Radiol. 2016;22:13-21.

30. Idilman IS, Keskin O, Celik A, Savas B, Elhan AH, Idilman R, et al. A comparison of liver fat content as determined by magnetic resonance imaging-proton density fat fraction and MRS versus liver histology in non-alcoholic fatty liver disease. Acta Radiol. 2016;57:271-8.

31. Guiu B, Petit JM, Loffroy R, Ben Salem D, Aho S, Masson D, et al. Quantification of liver fat content: comparison of tripleecho chemical shift gradient-echo imaging and in vivo proton MR spectroscopy. Radiology. 2009;250:95-102.

32. Chabanova E, Bille DS, Thisted E, Holm JC, Thomsen HS. 1H MRS assessment of hepatic steatosis in overweight children and adolescents: comparison between 3T and open 1T MR-systems. Abdom Imaging. 2013;38:315-9.

33. Kobayashi J, Tadokoro N, Watanabe M, Shinomiya M. A novel method of measuring intra-abdominal fat volume using helical computed tomography. Int J Obes Relat Metab Disord. 2002;26:398-402.

34. Yoshizumi T, Nakamura T, Yamane M, Islam AH, Menju M, Yamasaki K, et al. Abdominal fat: standardized technique for measurement at CT. Radiology. 1999;211:283-6.

35. Tamura S, Shimomura I. Contribution of adipose tissue and de novo lipogenesis to nonalcoholic fatty liver disease. J Clin Invest. 2005;115:1139-42.

36. Dasarathy S, Dasarathy J, Khiyami A, Joseph R, Lopez R, McCullough AJ. Validity of real time ultrasound in the diagnosis of hepatic steatosis: a prospective study. J Hepatol. 2009;51:1061-7.

37. Ratziu V, Charlotte F, Heurtier A, Gombert S, Giral P, Bruckert $\mathrm{E}$, et al. Sampling variability of liver biopsy in non-alcoholic fatty liver disease. Gastroenterology. 2005;128:1898-906.

38. Pickhardt PJ, Park SH, Hahn L, Lee SG, Bae KT, Yu ES. Specificity of unenhanced CT for non-invasive diagnosis of hepatic steatosis: implications for the investigation of the natural history of incidental steatosis. Eur Radiol. 2012;22:1075-82.

39. Saadeh S, Younossi ZM, Remer EM, Gramlich T, Ong JP, Hurley $\mathrm{M}$, et al. The utility of radiological imaging in nonalcoholic fatty liver disease. Gastroenterology. 2002;123:745-50.

40. Roldan-Valadez E, Favila R, Martinez-Lopez M, Uribe M, Méndez-Sánchez N. Imaging techniques for assessing hepatic fat content in nonalcoholic fatty liver disease. Ann Hepatol. 2008;7:212-20. 
41. Hayashi T, Saitoh S, Takahashi J, Tsuji Y, Ikeda K, Kobayashi M, et al. Hepatic fat quantification using the two-point Dixon method and fat color maps based on non-alcoholic fatty liver disease activity score. Hepatol Res. 2017;47:455-64.

42. Kuchay MS, Krishan S, Mishra SK, Farooqui KJ, Singh MK, Wasir JS. Effect of empagliflozin on liver fat in patients with type 2 diabetes and nonalcoholic fatty liver disease: a randomized controlled trial (E-LIFT Trial). Diabetes Care. 2018;41:1801-8.

43. Sumida Y, Murotani K, Saito M, Tamasawa A, Osonoi Y, Yoneda M. Effect of luseogliflozin on hepatic fat content in type 2 diabetes patients with non-alcoholic fatty liver disease: a prospective, single-arm trial (LEAD trial). Hepatol Res. 2019;49:64-71.

44. Joy TR, McKenzie CA, Tirona RG, Summers K, Seney S, Chakrabarti S, et al. Sitagliptin in patients with non-alcoholic steatohepatitis: a randomized, placebo-controlled trial. World J Gastroenterol. 2017;23(1):141-50.

45. Cui J, Philo L, Nguyen P, Hofflich H, Hernandez C, Bettencourt R, et al. Sitagliptin vs. placebo for non-alcoholic fatty liver disease: a randomized controlled trial. J Hepatol. 2016;65:369-76.

46. Musso G, Cassader M, Rosina F, Gambino R. Impact of current treatments on liver disease, glucose metabolism and cardiovascular risk in non-alcoholic fatty liver disease (NAFLD): a systematic review and meta-analysis of randomized trials. Diabetologia. 2012;55(4):885-904.

47. Promrat K, Kleiner DE, Niemeier HM, Jackvony E, Kearns M, Wands JR, et al. Randomized controlled trial testing the effects of weight loss on nonalcoholic steatohepatitis. Hepatology. 2010;51:121-9.

48. Armstrong MJ, Houlihan DD, Rowe IA, Clausen WH, Elbrond B, Gough SC, et al. Safety and efficacy of liraglutide in patients with type 2 diabetes and elevated liver enzymes: individual patient data meta-analysis of the LEAD program. Aliment Pharmacol Ther. 2013;37:234-42.

49. Cusi K, Sattar N, Garcia-Perez LE, Pavo I, Yu M, Robertson KE, et al. Dulaglutide decreases plasma aminotransferases in people with type 2 diabetes in a pattern consistent with liver fat reduction: a post hoc analysis of the AWARD programme. Diabet Med. 2018;35:1434-9.

50. Dutour A, Abdesselam I, Ancel P, Kober F, Mrad G, Darmon P, et al. Exenatide decreases liver fat content and epicardial adipose tissue in patients with obesity and type 2 diabetes: a prospective randomized clinical trial using magnetic resonance imaging and spectroscopy. Diabetes Obes Metab. 2016;18:882-91.

51. Dasarathy S, Merli M. Sarcopenia from mechanism to diagnosis and treatment in liver disease. J Hepatol. 2016;65:1232-44.

52. Lee YH, Kim SU, Song K, Park JY, Kim DY, Ahn SH, et al. Sarcopenia is associated with significant liver fibrosis independently of obesity and insulin resistance in nonalcoholic fatty liver disease: nationwide surveys (KNHANES 2008-2011). Hepatology. 2016;63:776-86.

53. Koo BK, Kim D, Joo SK, Kim JH, Chang MS, Kim BG, et al. Sarcopenia is an independent risk factor for non-alcoholic steatohepatitis and significant fibrosis. J Hepatol. 2017;66:123-31.

54. Mizuno N, Seko Y, Kataoka S, Okuda K, Furuta M, Takemura $M$. Increase in the skeletal muscle mass to body fat mass ratio predicts the decline in transaminase in patients with nonalcoholic fatty liver disease. J Gastroenterol. 2019;54:160-70.

55. Bolinder J, Ljunggren Ö, Kullberg J, Johansson L, Wilding J, Langkilde AM, et al. Effects of dapagliflozin on body weight, total fat mass, and regional adipose tissue distribution in patients with type 2 diabetes mellitus with inadequate glycemic control on metformin. J Clin Endocrinol Metab. 2012;97:1020-31.

56. Tosaki T, Kamiya H, Himeno T, Kato Y, Kondo M, Toyota K, et al. Sodium-glucose co-transporter 2 inhibitors reduce the abdominal visceral fat area and may influence the renal function in patients with type 2 diabetes. Intern Med. 2017;56(6):597-604.
57. Sasaki T, Sugawara M, Fukuda M. Sodium-glucose cotransporter 2 inhibitor-induced changes in body composition and simultaneous changes in metabolic profile: 52 -week prospective LIGHT (Luseogliflozin: the Components of Weight Loss in Japanese Patients with Type 2 Diabetes Mellitus) Study. J Diabetes Investig. 2019;10:108-17.

58. Boyko EJ, Fujimoto WY, Leonetti DL, Newell-Morris L. Visceral adiposity and risk of type 2 diabetes: a prospective study among Japanese Americans. Diabetes Care. 2000;23(4):465-71.

59. Nakamura T, Tokunaga K, Shimomura I, Nishida M, Yoshida S, Kotani K, et al. Contribution of visceral fat accumulation to the development of coronary artery disease in non-obese men. Atherosclerosis. 1994;107(2):239-46.

60. Pouliot MC, Despres JP, Nadeau A, Moorjani S, Prud'Homme $\mathrm{D}$, Lupien PJ, et al. Visceral obesity in men: associations with glucose tolerance, plasma insulin, and lipoprotein levels. Diabetes. 1992;41(7):826-34.

61. Yusuf S, Hawken S, Ounpuu S, Bautista L, Franzosi MG, Commerford $\mathrm{P}$, et al. Obesity and the risk of myocardial infarction in 27,000 participants from 52 countries: a case-control study. Lancet. 2005;366:1640-9.

62. Fujimoto WY, Bergstrom RW, Boyko EJ, Chen KW, Leonetti DL, Newell-Morris L, et al. Visceral adiposity and incident coronary heart disease in Japanese-American men: the 10-year follow-up results of the Seattle Japanese-American Community Diabetes Study. Diabetes Care. 1999;22:1808-12.

63. Obata A, Kubota N, Kubota T, Iwamoto M, Sato H, Sakurai Y, et al. Tofogliflozin improves insulin resistance in skeletal muscle and accelerates lipolysis in adipose tissue in male mice. Endocrinology. 2016;157(3):1029-42.

64. Maruyama C, Imamura K, Teramoto T. Assessment of LDL particle size by triglyceride/HDL-cholesterol ratio in non-diabetic, healthy subjects without prominent hyperlipidemia. J Atheroscler Thromb. 2003;10:186-91.

65. St-Pierre AC, Cantin B, Dagenais GR, Mauriège P, Bernard PM, Després JP, et al. Low-density lipoprotein subfractions and the long term risk of ischemic heart disease in men: 13-year follow-up data from the Québec Cardiovascular Study. Arterioscler Thromb Vasc Biol. 2005;25:553-9.

66. Hayashi T, Fukui T, Nakanishi N, Yamamoto S, Tomoyasu M, Osamura A, et al. Dapagliflozin decreases small dense low-density lipoprotein-cholesterol and increases high-density lipoprotein 2-cholesterol in patients with type 2 diabetes: comparison with sitagliptin. Cardiovasc Diabetol. 2017;16:8.

67. Zoppini G, Targher G, Negri C, Stoico V, Perrone F, Muggeo M, et al. Elevated serum uric acid concentrations independently predict cardiovascular mortality in type 2 diabetic patients. Diabetes Care. 2009;32(9): 1716-20.

68. Bjornstad P, Lanaspa MA, Ishimoto T, Kosugi T, Kume S, Jalal D, et al. Fructose and uric acid in diabetic nephropathy. Diabetologia. 2015;58(9):1993-2002.

69. Cheeseman C. Solute carrier family 2, member 9 and uric acid homeostasis. Curr Opin Nephrol Hypertens. 2009;18(5):428-32.

70. Tahara A, Takasu T, Yokono M, Imamura M, Kurosaki E. Characterization and comparison of SGLT2 inhibitors: Part 3. Effects on diabetic complications in type 2 diabetic mice. Eur J Pharmacol. 2017;809:163-71.

71. Shyangdan DS, Uthman OA, Waugh N. SGLT-2 receptor inhibitors for treating patients with type 2 diabetes mellitus: a systematic review and network meta-analysis. BMJ Open. 2016;6(2):e009417.

72. Watanabe S, Hashimoto E, Ikejima K, Uto H, Ono M, Sumida Y, et al. Evidence based clinical practice guidelines for nonalcoholic fatty liver disease/nonalcoholic steatohepatitis. J Gastroenterol. 2015;50:364-77. 
73. Dulai PS, Singh S, Patel J, Soni M, Prokop LJ, Younossi Z, et al. Increased risk of mortality by fibrosis stage in nonalcoholic fatty liver disease: systematic review and meta-analysis. Hepatology. 2017;65:1557-65.

74. Nakahara T, Hyogo H, Yoneda M, Sumida Y, Eguchi Y, Fujii $\mathrm{H}$. Type 2 diabetes mellitus is associated with the fibrosis severity in patients with nonalcoholic fatty liver disease in a large retrospective cohort of Japanese patients. J Gastroenterol. 2014;49(11):1477-84.

75. Ito D, Shimizu S, Inoue K, Saito D, Yanagisawa M, Inukai K, et al. Comparison of ipragliflozin and pioglitazone effects on nonalcoholic fatty liver disease in patients with type 2 diabetes: a randomized, 24-week, open-label, active controlled trial. Diabetes Care. 2017;40:1364-72.

76. Itani $\mathrm{T}$, Ishihara T. Efficacy of canagliflozin against non-alcoholic fatty liver disease: a prospective cohort study. Obes Sci Pract. 2018;4:477-82.
77. Ohki T, Isogawa A, Toda N, Tagawa K. Effectiveness of ipragliflozin, a sodiumglucose co-transporter 2 inhibitor, as a second-line treatment for non-alcoholic fatty liver disease patients with type 2 diabetes mellitus who do not respond to incretin-based therapies including glucagon-like peptide-1 analogs and dipeptidyl peptidase-4 inhibitors. Clin Drug Investig. 2016;36:313-9.

78. Inoue M, Hayashi A, Taguchi T, Arai R, Sasaki S, Takano K, et al. Effects of canagliflozin on body composition and hepatic fat content in type 2 diabetes patients with non-alcoholic fatty liver disease. J Diabetes Investig. 2018. https://doi.org/10.1111/ jdi.12980.

79. Akuta N, Kawamura Y, Watanabe C, Nishimura A, Okubo M, Mori Y, et al. Impact of SGLT2 inhibitor to histological features and glucose metabolism of non-alcoholic fatty liver disease complicated by diabetes mellitus. Hepatol Res. 2018. https://doi. org/10.1111/hepr.13304. 